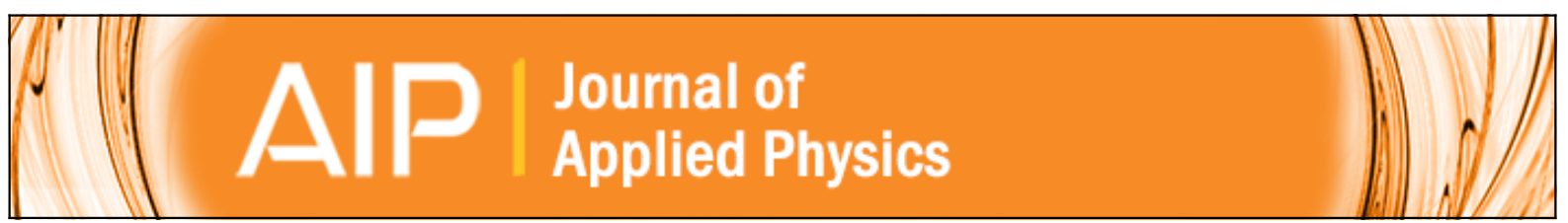

Broadening of $\mathrm{x}$-ray diffraction lines from small subgrains containing gradients of spacing

C. R. Houska

Citation: Journal of Applied Physics 49, 2991 (1978); doi: 10.1063/1.325147

View online: http://dx.doi.org/10.1063/1.325147

View Table of Contents: http://scitation.aip.org/content/aip/journal/jap/49/5?ver=pdfcov

Published by the AIP Publishing

Articles you may be interested in

Broadening of $\mathrm{x}$-ray powder diffraction lines under nonhydrostatic stress

J. Appl. Phys. 82, 142 (1997); 10.1063/1.365792

X-ray diffraction from d spacing gradients along ion-implanted zones

J. Appl. Phys. 69, 8096 (1991); 10.1063/1.347459

Line-broadening analysis of synchrotron $\mathrm{x}$-ray diffraction data

J. Appl. Phys. 61, 2813 (1987); 10.1063/1.337872

X-Ray Diffraction from Small Crystallites

J. Appl. Phys. 35, 1718 (1964); 10.1063/1.1713726

X-Ray Diffraction Line Broadening and Strain Hardening

J. Appl. Phys. 20, 1257 (1949); 10.1063/1.1698320

MIT LINCOLN

LABORATORY CAREERS

Discover the satisfaction of innovation and service

to the nation
- Space Control

- Air \& Missile Defense

- Communications Systems \& Cyber Security

- Intelligence, Surveillance and Reconnaissance Systems

- Advanced
Electronics
- Tactical Systems
- Homeland
Protection
- Air Traffic Control

- Air Traffic Control

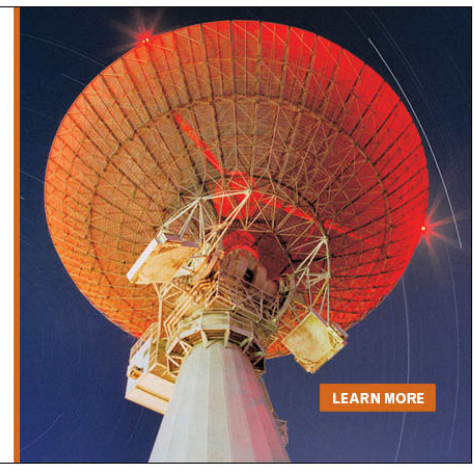




\title{
Broadening of $x$-ray diffraction lines from small subgrains containing gradients of spacing
}

\author{
C. R. Houska \\ Department of Materials Engineering, Virginia Polytechnic Institute and State University, Blacksburg, \\ Virginia 24061 \\ (Received 2 September 1977; accepted for publication 1 December 1977)
}

\begin{abstract}
Recent data on subgrain size and spacing gradients in the $\mathrm{Cu}-\mathrm{Ni}$ system allow a more critical examination of a technique currently used to obtain the composition profiles of small diffusion zones. These data support the assumption that the broadening from individual subgrains need not include a spacing gradient term when volume diffusion is predominant. However, care should be taken in the interpretation of the earliest stage of diffusion where grain boundaries, surface, and other defects may introduce large spacing gradients.
\end{abstract}

PACS numbers: $61.10 . \mathrm{Dp}, 61.70 . \mathrm{Wp}, 61.70 . \mathrm{Ng}$

$\mathrm{X}$-ray diffraction has been found to give accurate composition profiles for small diffusion zones extending over several $\mu \mathrm{m} .{ }^{1-9}$ This approach is based upon the slowly varying long-range spacing change associated with diffusion samples. More recently, this has been applied to the long-range interface strains between dissimilar materials generated after cooling or heating. ${ }^{10}$ The latter strain can be attributed to differences in thermal expansion or contraction between dissimilar materials. At the time the initial theoretical paper was published, very little was known about defect structures generated along diffusion zones. Since this paper, data were obtained on subgrain size along with data on the more usual spacing (or composition) gradient as a result of further refinements of the technique. ${ }^{9}$ At this time, a better understanding is possible concerning the validity of the models originally proposed. ${ }^{1}$ One model treated the diffraction from diffusion zones containing a sufficiently large number of small subgrains so that the spacing gradient through any one is negligible. In this case (A), the line broadening due to a small subgrain size is dominant, and broadening due to spacing changes within a single subgrain can be neglected. At the other extreme, a simple expression is obtained if both subgrain size and the spacing gradient are large. In this case (B), the line broadening from individual subgrains is due almost entirely to the spacing change. A linear element approximation was employed to arrive at both models as limiting forms. Model A, which allows the spacing gradient to be neglected over individual subgrains, has been used exclusively in our work and is believed to be appropriate for volume diffusion. Here, the overall composition change is represented by a large number of subgrains with small steps in composition (or $d$ spacing) between neighboring subgrains. Although each subgrain may give diffraction lines that are broadened by small size and localized nonuniform strain, no experimental evidence was found for the existence of nonuniform strain broadening in the $\mathrm{Cu}-\mathrm{Ni}, \mathrm{Cu}-\mathrm{Ag}$, and $\mathrm{Au}-\mathrm{Pd}$ systems. This may not be so for the earliest stages of diffusion. For metal films on $\mathrm{Si}$, long-range interface strain could be important for most stages of reaction after cooling from the reaction temperature to lower temperatures.

This paper presents an extension of case A giving a mathematical form for combined size and some spacing gradient broadening. It also provides a test on the acceptable range over which model $A$ can be safely applied.

An $\mathrm{x}$-ray intensity band may be represented by the superposition of intensity equations having the form ${ }^{1}$

$$
\frac{\phi}{N_{3}}=\sum_{-\left(N_{3}-1\right)}^{N_{3}-1}\left(\frac{L_{n}}{N_{3}}\right) \exp \left(2 \pi i n h_{3}\right)
$$

where

$$
\frac{L_{n}}{N_{3}}=\frac{\sin \pi l\left(\Delta d / d_{0}\right) n\left(1-|n| / N_{3}\right)}{\pi l\left(\Delta d / d_{0}\right) n},
$$

$N_{3}$ is the number of layers in a subgrain with reflecting planes of a minimum spacing $d_{0}$ and a maximum of $d_{0}+\Delta d, l$ is the order of reflection, and the sum is taken over all pairs of planes $n$ up to a maximum restricted by the total number of planes $N_{3}$. The reciprocal lattice vector is given by $h_{3} b_{3}$ with $\left|b_{3}\right|$ $=1 /\langle d\rangle$. It has not been possible to carry out the summation directly in the general case. However, Eq. (1) can be transformed into the more convenient integral form which does give a useful result:

$$
\frac{\Phi}{N_{3}^{2}}=2 \int_{0}^{1} \frac{\sin \pi S u(1-u)}{\pi S u} \cos \left(2 \pi u h_{3}^{0}\right) d u
$$

where $u=n / N_{3}, S=l\left(\Delta d / d_{0}\right) N_{3}$, and $h_{3}^{0}=N_{3} h_{3}$. This integral was first used by Mittemeijer and Delhez ${ }^{11}$ for examining the decrease in peak intensity due to an increase in $\Delta d / d_{0}$. However, no analytic expression was given for the shape of the cosine transform. If the Fourier coefficient is expanded and only the first term is retained, this gives case $A$, i. e. ,

$$
L_{u} / N_{3} \rightarrow 1-u \text {. }
$$

Furthermore, the Fourier coefficient can be expressed as

$$
L_{u} / N_{3}=(1-u)\left[1-F\left(S^{2}, u\right)\right]
$$

where $F\left(S^{2}, u\right)$ is a Fourier series with coefficients containing the parameter $S^{2}$ and $u$. A usable transform 


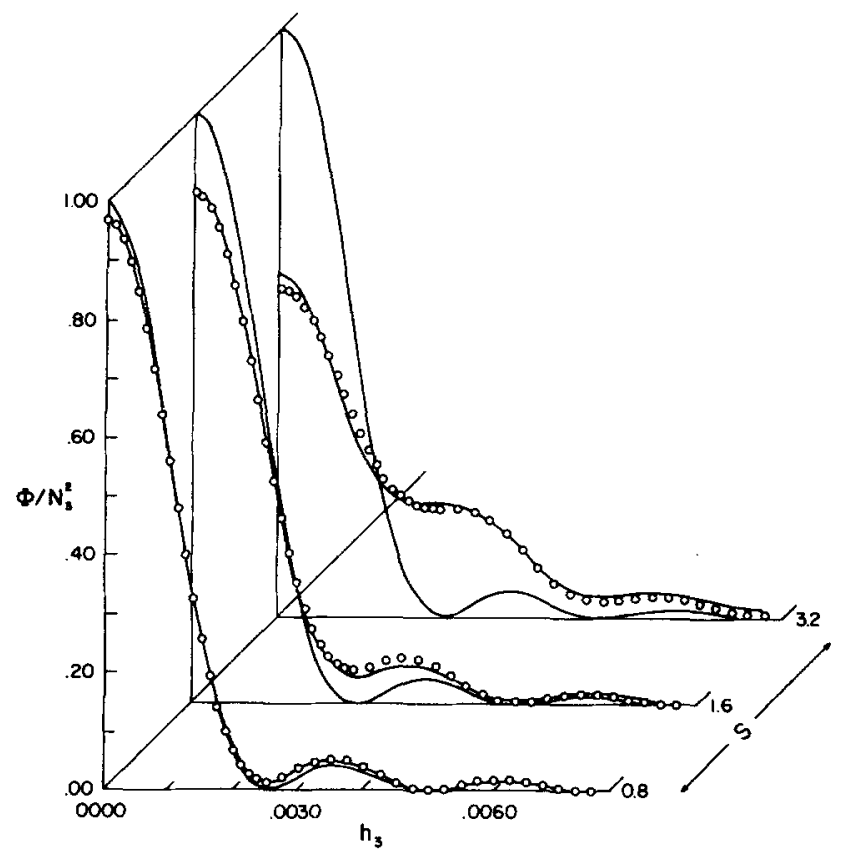

FIG. 1. Line shapes for a subgrain having 400 layers with $S=0.8,1.6$, and 3.2. Three identical normalized curves with only particle size broadening are included for reference $(1.0$ at $h_{3}=0$ ). The lower solid curves (at $h_{3}=0$ ) were calculated using Eq. (6), while the points were calculated from the series given by $\mathrm{Eq}$. (1).

is obtained from the first term of the Fourier expansion given by

$$
F\left(S^{2}, u\right)=K S^{2} \sin \pi u,
$$

where $K=$ const. If Eq. (4) is substituted into Eq. (2) and the transform is taken, one obtains

$$
\frac{\Phi}{N_{3}^{2}}=\left(\frac{\sin \pi h_{3}^{0}}{\pi h_{3}^{0}}\right)^{2}-K S^{2} f\left(h_{3}^{0}\right)
$$

where

$$
\begin{aligned}
f\left(h_{3}^{0}\right)= & \frac{\sin ^{2} \pi\left(\frac{1}{2}-h_{3}^{0}\right)}{\pi\left(\frac{1}{2}-h_{3}^{0}\right)}+\frac{\sin ^{2} \pi\left(\frac{1}{2}+h_{3}^{0}\right)}{\pi\left(\frac{1}{2}+h_{3}^{0}\right)}+\frac{\sin 2 \pi\left(\frac{1}{2}-h_{3}^{0}\right)}{\left[2 \pi\left(\frac{1}{2}-h_{3}^{0}\right)\right]^{2}} \\
& -\frac{\cos 2 \pi\left(\frac{1}{2}-h_{3}^{0}\right)}{2 \pi\left(\frac{1}{2}-h_{3}^{0}\right)}+\frac{\sin 2 \pi\left(\frac{1}{2}+h_{3}^{0}\right)}{\left[2 \pi\left(\frac{1}{2}+h_{3}^{0}\right)\right]^{2}}-\frac{\cos 2 \pi\left(\frac{1}{2}+h_{3}^{0}\right)}{2 \pi\left(\frac{1}{2}+h_{3}^{0}\right)}
\end{aligned}
$$

In this approximation, the term $K S^{2} f\left(h_{3}^{0}\right)$ is sufficiently small so that the following equation can also be used:

$$
\frac{\Phi}{N_{3}^{2}}=\left(\frac{\sin \pi h_{3}^{0}}{\pi h_{3}^{0}}\right)^{2}+\exp \left[-K S^{2} f\left(h_{3}^{0}\right)\right]-1
$$

which gives a slightly better fit to Eq. (1). At this point, it becomes evident that the first term expansion of $F\left(S^{2}, u\right)$ gives six terms after taking the cosine transform. Although greater accuracy for larger values of $S$ could be obtained by introducing additional Fourier terms, this no longer leads to a convenient result because of the large number of terms generated.
Figure 1 contains three identical reference plots for the pure particle size case with $N_{3}=400$ and $\Delta d / d_{0}=0$ along with plots of Eqs. (1) and (6) with $N_{3}=400$ and $S=0.8,1.6$, and 3.2. It can be seen that for $S=0.8$ the difference between these and the reference curve is at the threshold of being measurable. For $S=1.6$ and 3.2 , the influence of spacing gradient on line shape becomes obvious. Clearly, the oscillations no longer go to zero, and tails develop. In an actual diffusion sample, one would find a distribution of subgrain sizes, at each composition, that would smooth out the oscillations. Equation (6) should not be used for $S>3.2$ because only one Fourier term in Eq. (4) is no longer valid. For larger values of $S$, Eq. (1) should be used.

There are sufficient data from the $\mathrm{Cu}-\mathrm{Ni}$ system to determine the importance of spacing gradient broadening. Two stages of diffusion have been reported for this system. For short times, grain boundary, surface, and pipe diffusion are important. ${ }^{49}$ However, at longer times, the interdiffusion of a $\mathrm{Ni}$ film of several $\mu \mathrm{m}$ into a thick substrate can be treated as a one-dimensional volume diffusion problem. ${ }^{9}$ In this case, the diffusion zone extends over several $\mu \mathrm{m}$, and the subgrain sizes along this zone range from 334 to $790 \AA$ at the extreme ends of the diffusion zone. To determine whether $S<0.8$ for this case, consider the diffusion zone to be $3 \mu \mathrm{m}$ and the composition to range from pure $\mathrm{Cu}(a$ $=3.6153 \AA)$ to pure $\mathrm{Ni}(a=3.5238 \AA)$. This would give a fractional change in $d$ spacing of $2.56 \times 10^{-2} / N_{s}$ per subgrain if $N_{s}$ equals the number of subgrains within the zone. Consequently, $S=l(\Delta d / d)\left(N_{3} / N_{s}\right)$ which can be written as $S=l\left(\Delta d / d^{2}\right)\left(L^{2} / Z S\right)$ with a subgrain size $L=N_{3} d$ and a zone size $Z S=N_{S} L$. The $111(l=1)$ and $222(l=2)$ intensity bands were examined in the $\mathrm{CuNi}$ studies. By using these values, one obtains $L$ values of 1388 and $982 \AA$ for these bands which are larger than the range of experimental values. Consequently, the inequality $S_{\text {exp }}<0.8$ is satisfied. This estimate is valid for the central portion of the diffusion zone where the spacing gradient is largest. At the end regions of a diffusion zone, rounding of the composition profile gives a smaller average spacing gradient which makes the estimated $S_{\text {exp }}$ even smaller. A smaller subgrain size allows more subgrains to be distributed along the diffusion zone with each experiencing a smaller overall range in composition. These conservative estimates indicate that for volume diffusion in the $\mathrm{Cu}-\mathrm{Ni}$ system the line broadening from individual subgrains is not influenced by spacing changes. It is possible that in the earliest stage of diffusion, in which grain boundary diffusion is found, significant broadening from spacing gradients may very well be present. In this case, there can be a large volume fraction of material with a significant spacing gradient. Consequently, model $\mathrm{A}$ which allows individual subgrains to assume a single composition may not be strictly valid. The possible influence of spacing gradient broadening should be examined under these extreme conditions.

The author is grateful to the National Science Foundation for financial assistance and to Tom Adler for providing the calculations required for Fig. 1. 
${ }^{1}$ C.R. Houska, J. Appl. Phys. 41, 69 (1970).

${ }^{2}$ D.R. Tenney, J.A. Carpenter, and C.R. Houska, J. Appl. Phys. 41, 4485 (1970).

${ }^{3}$ J.A. Carpenter, D.R. Tenney, and C.R. Houska, J. Appl. Phys. 42, 4305 (1971).

'J. Unnam, J. A. Carpenter, and C. R. Houska, J. Appl. Phys. 44, 1957 (1973).

${ }^{5}$ D. R. Tenney and P.K. Talty, Metall. Trans. 5, 241 (1974). ${ }^{6}$ R. Houska, Thin Solid Films 25, 451 (1975).
${ }^{7}$ M. Murakami and D. de Fontaine, J. Appl. Phys, 47, 2850 (1976); 47, 2857 (1976).

${ }^{8} \mathrm{~J}$. Unnam and C. R. Houska, J. Appl. Phys. 47, 4336 (1976). ${ }^{9}$ C. R. Houska, G. Subbaraman, and F. Dietrich, Thin Solid Films 44, 217 (1977).

${ }^{10}$ M. Murakami, IBM T.J. Watson Res. Center Research Report, RC6427 (No. 27720), 1977 (unpublished).

${ }^{11}$ E.J. Mittemijer and R. Delhez, J. Appl. Phys, 47, 1702 (1976). 